\title{
An NMR Tube Degradation Method for Sugar Analysis of Glycosides
}

José-Luis Giner, * Ju Feng and David J. Kiemle

\section{Supporting Information}

Figure S1: ${ }^{1} \mathrm{H}-\mathrm{NMR}$ spectra of the anomeric regions of sugars 5-35

pp 2-7

Figure S2: ${ }^{1} \mathrm{H}-\mathrm{NMR}$ spectra of the methyl regions of sugars 22-34

p 8

Figure S3: ${ }^{1} \mathrm{H}-\mathrm{NMR}$ spectrum of furfural

p 9

Figure S4: ${ }^{1} \mathrm{H}-\mathrm{NMR}$ spectra of sugars 5-7: heating timecourse

p 10

Figure S5: ${ }^{1} \mathrm{H}-\mathrm{NMR}$ spectra of sugars 5-7: different concentrations

Figure S6: ${ }^{1} \mathrm{H}-\mathrm{NMR}$ spectra of glucose (5) at different $\left[\mathrm{D}_{2} \mathrm{SO}_{4}\right]$

p 12

Figure S7: Graphed ${ }^{1} \mathrm{H}-\mathrm{NMR}$ signals of glucose (5) at different $\left[\mathrm{D}_{2} \mathrm{SO}_{4}\right]$

p 13

Figure S8: $T_{1}$ measurements of 5-7 and 18

p 14-16

Figure S9: ${ }^{1} \mathrm{H}-\mathrm{NMR}$ spectra of naringin (1) hydrolysis with DMSO

p 17

Figure S10: ${ }^{1} \mathrm{H}-\mathrm{NMR}$ spectra of glucose (5) at different [DMSO]

p 18 
Figure S1

p. 1 of 6

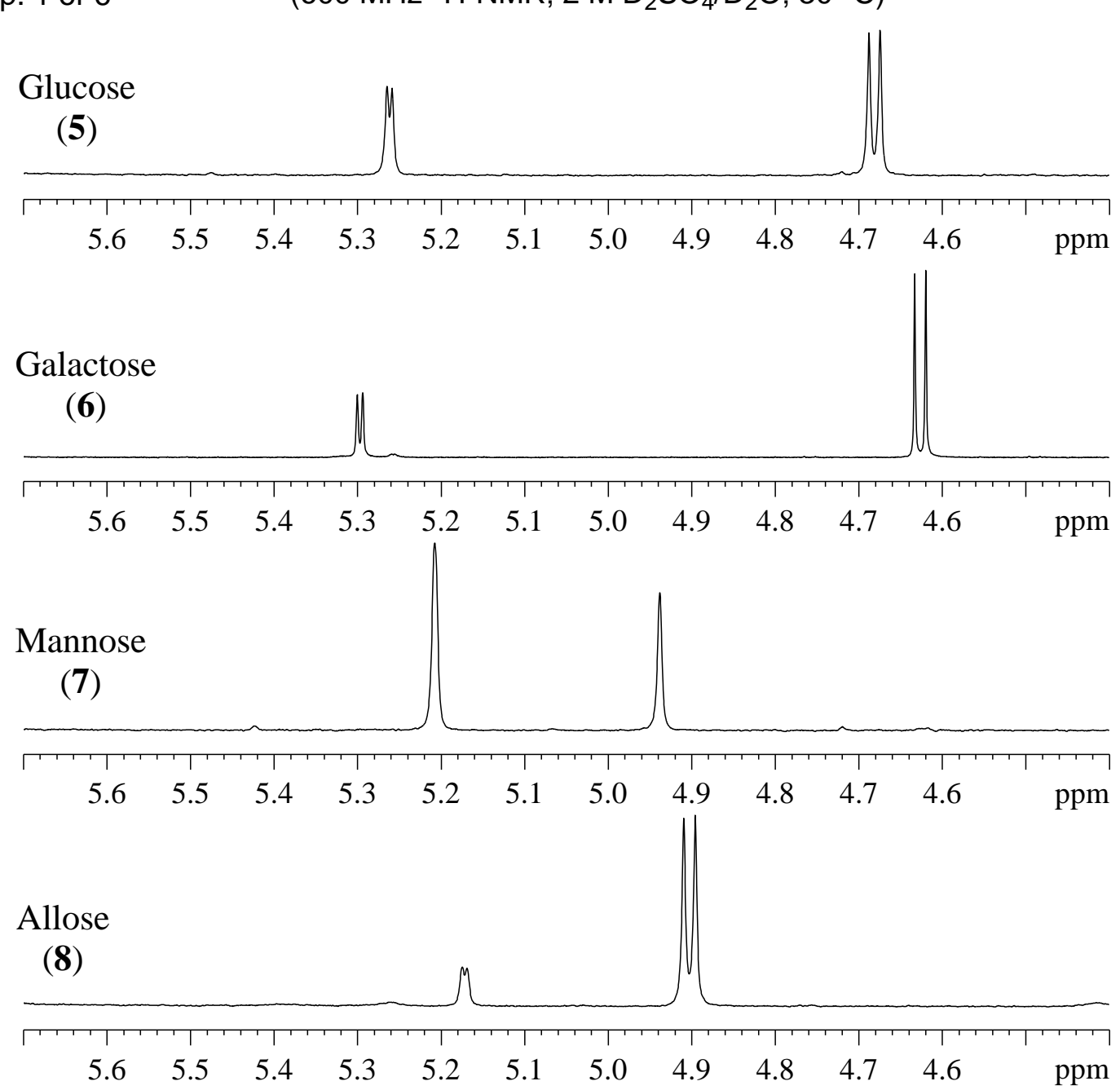

Allose (equilibrium)

(8)

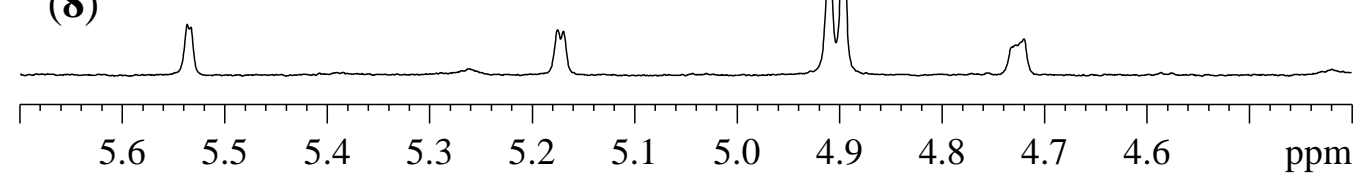

Altrose

(8)

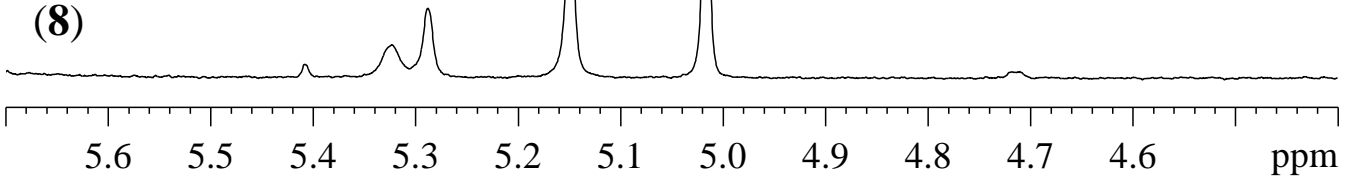


Figure S1

p. 2 of 6

Altrose (equilibrium)

(9)
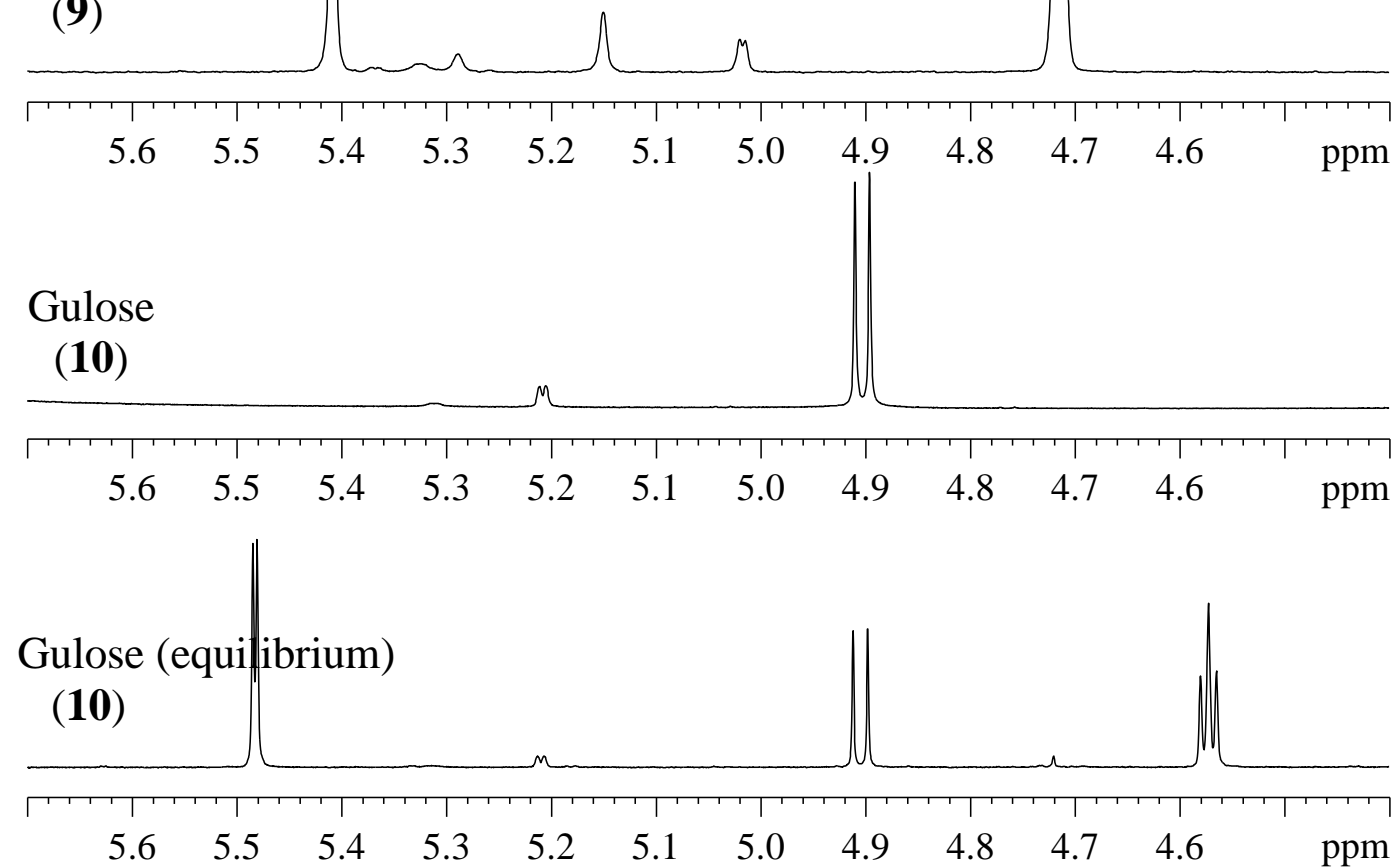

Idose

(11)

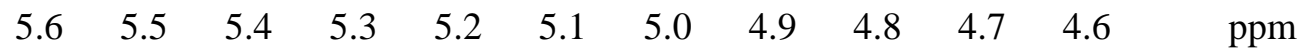

Idose (equilibrium)

(11)

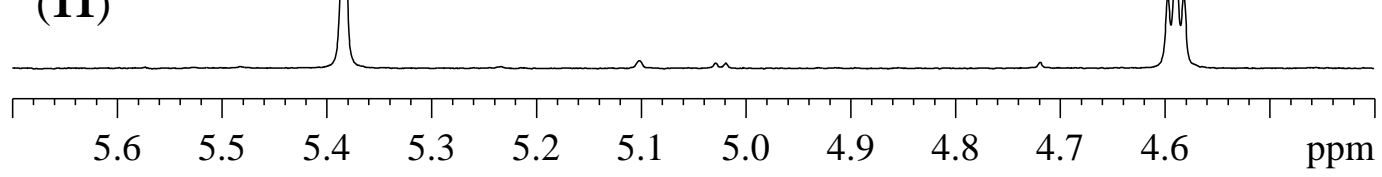

Talose

(12)

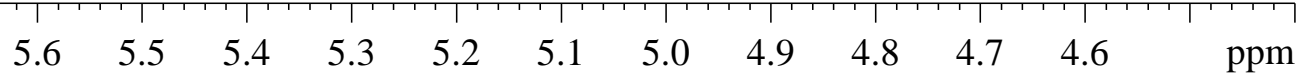


Figure S1

p. 3 of 6

Glucuronic acid

(13)
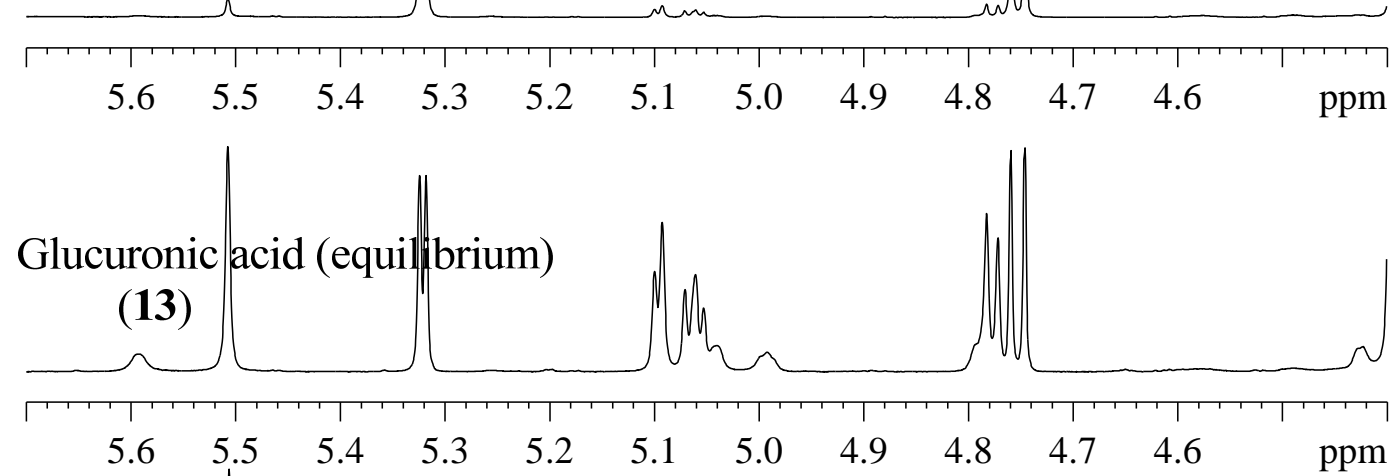

Glucuronolactone
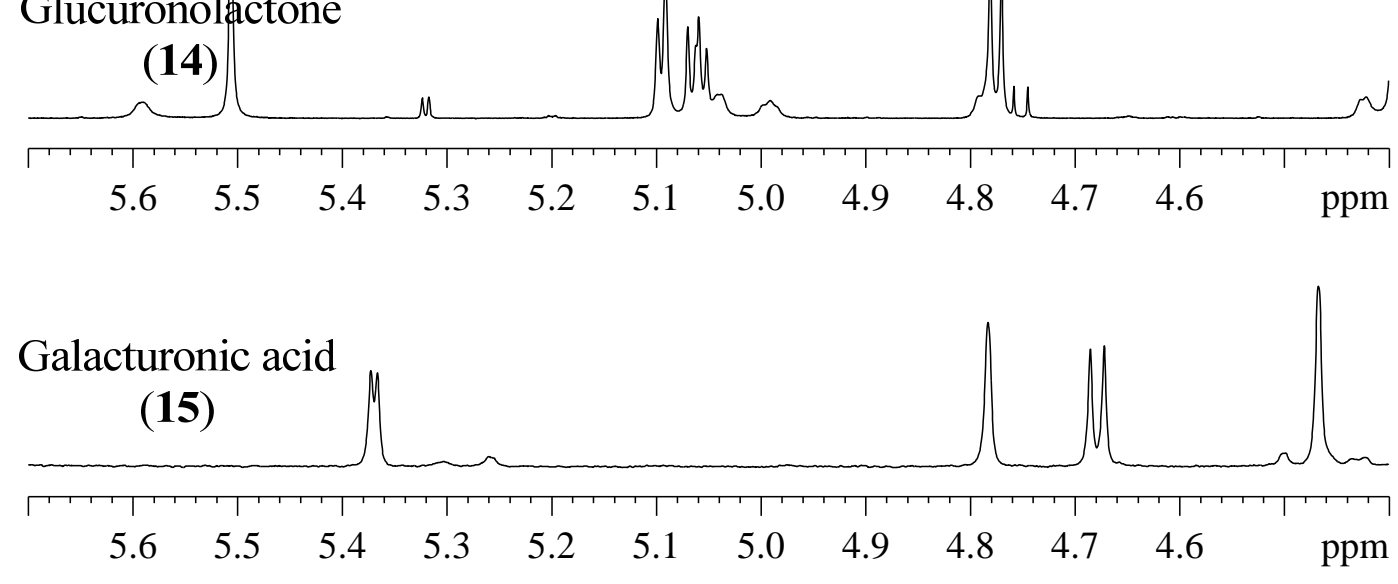

Arabinose

(16)

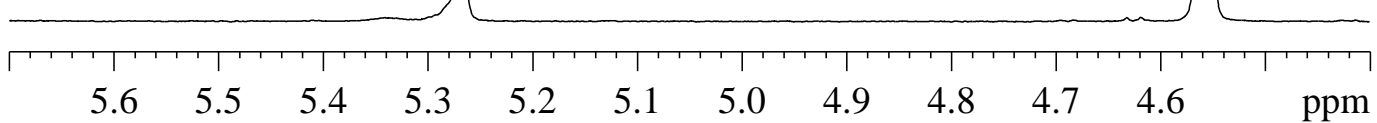

Ribose

(17)

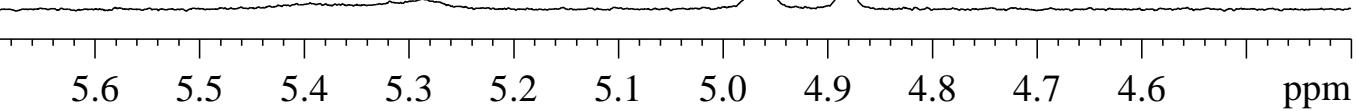


Figure S1

p. 4 of 6

Xylose

(18)

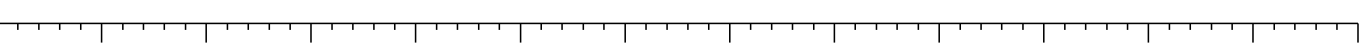

$\begin{array}{llllllllllll}5.6 & 5.5 & 5.4 & 5.3 & 5.2 & 5.1 & 5.0 & 4.9 & 4.8 & 4.7 & 4.6 & \mathrm{ppm}\end{array}$

Lyxose

(19)
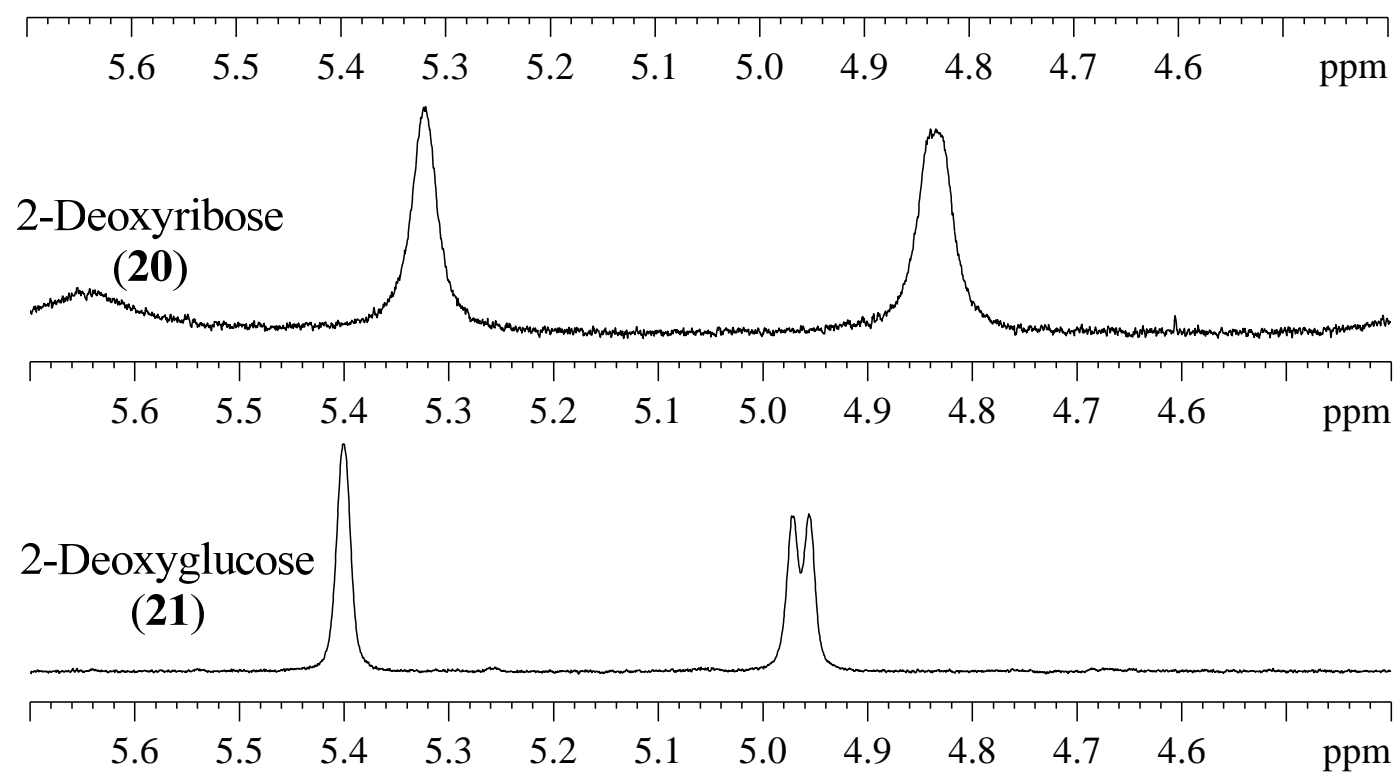

Fucose

(22)

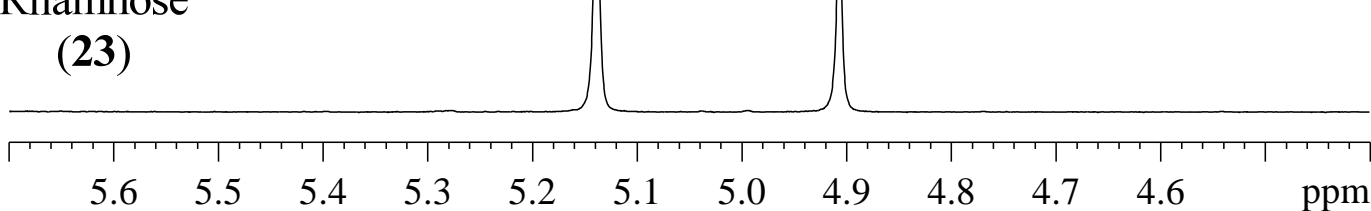


Figure S1

p. 5 of 6

Quinovose

(24)
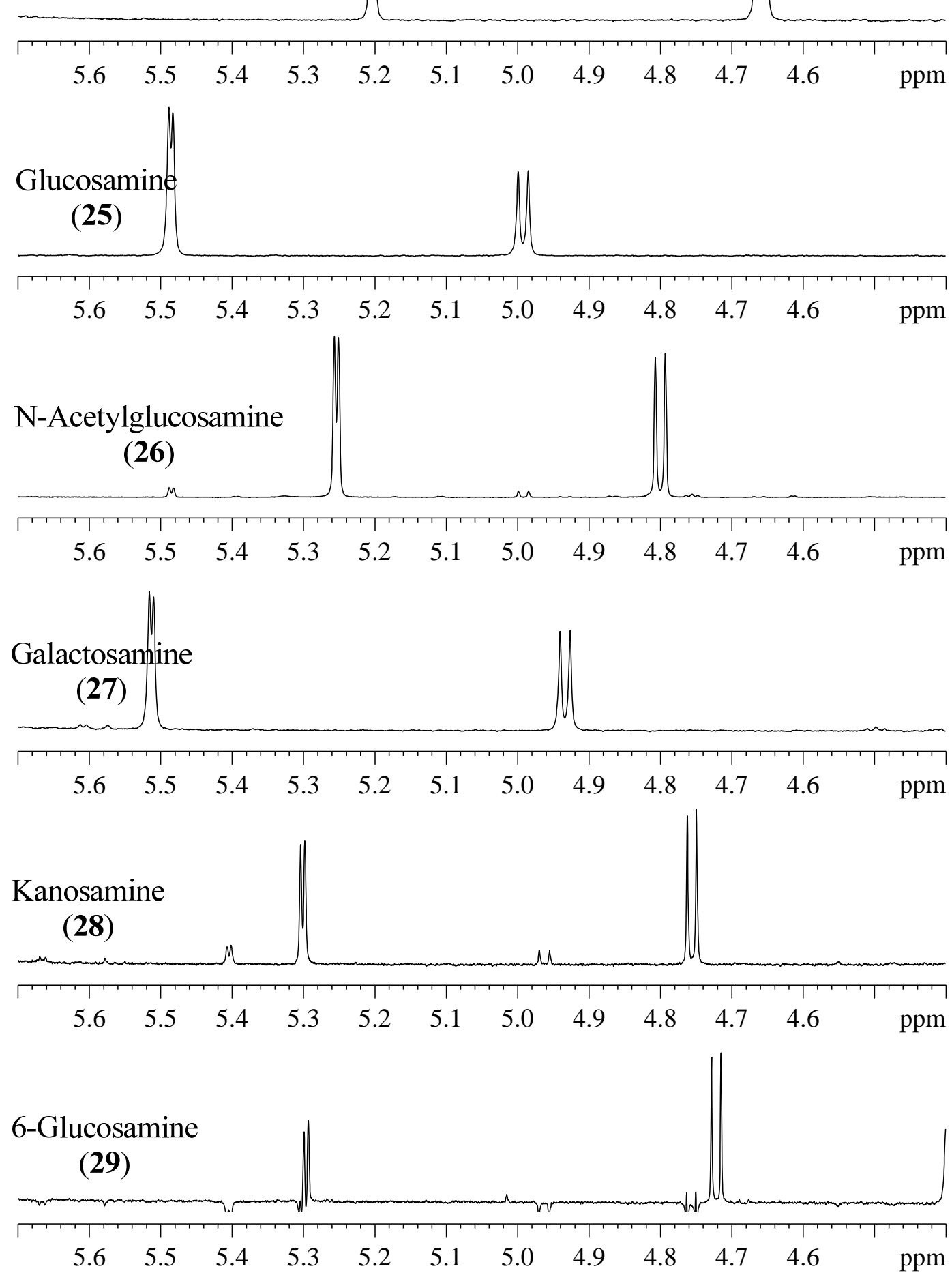
Figure S1

p. 6 of 6

Nebrosamine

(30) (3)

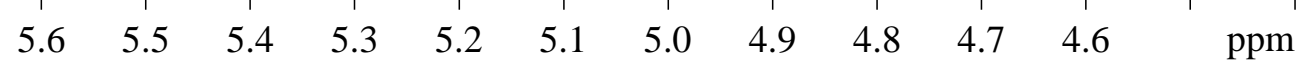

Cellobiose

(31)
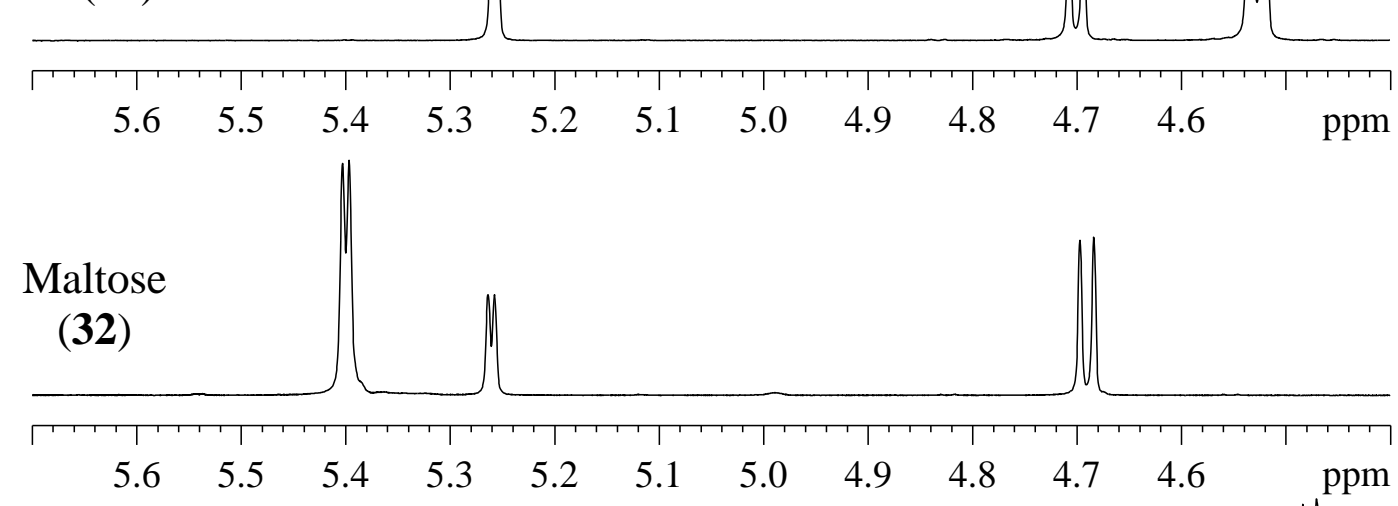

Lactose

(33)

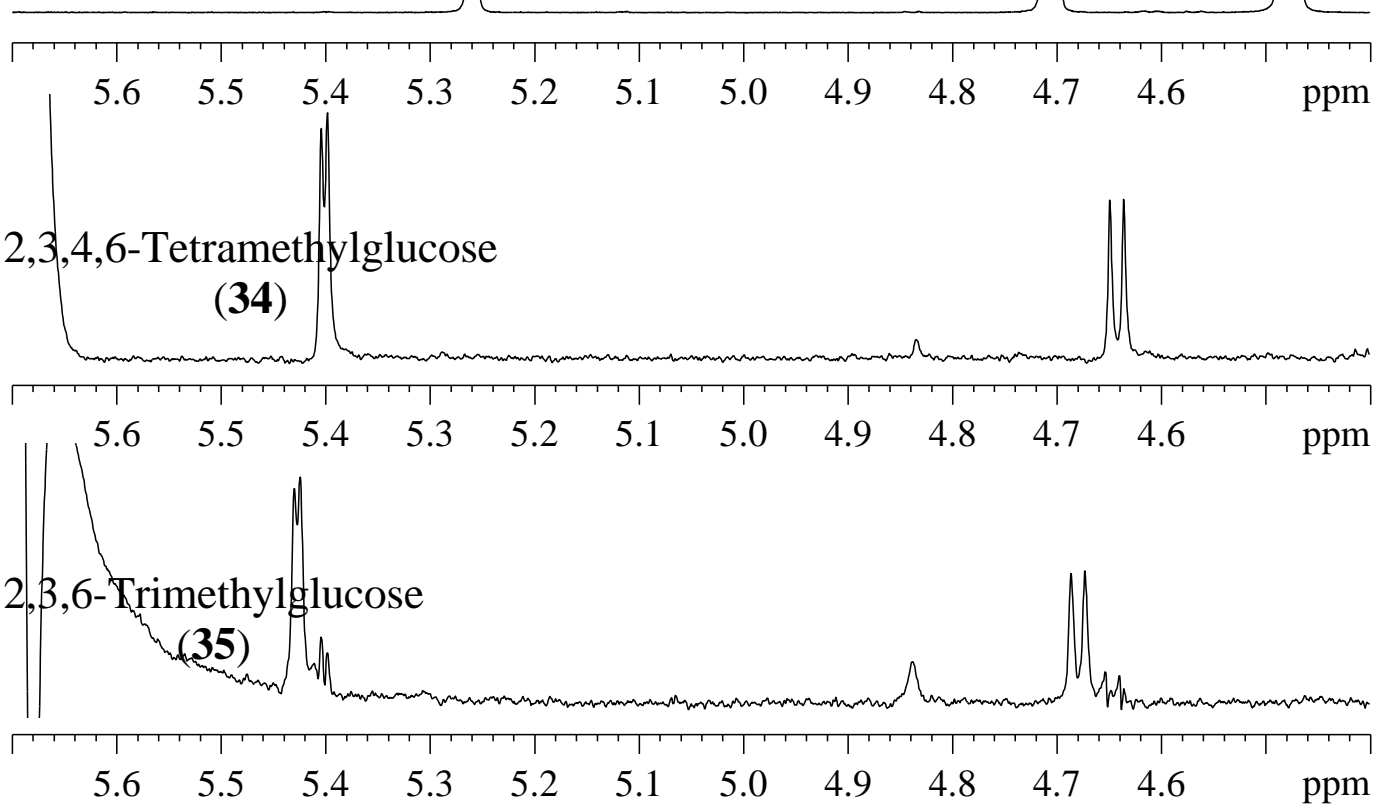


Figure S2

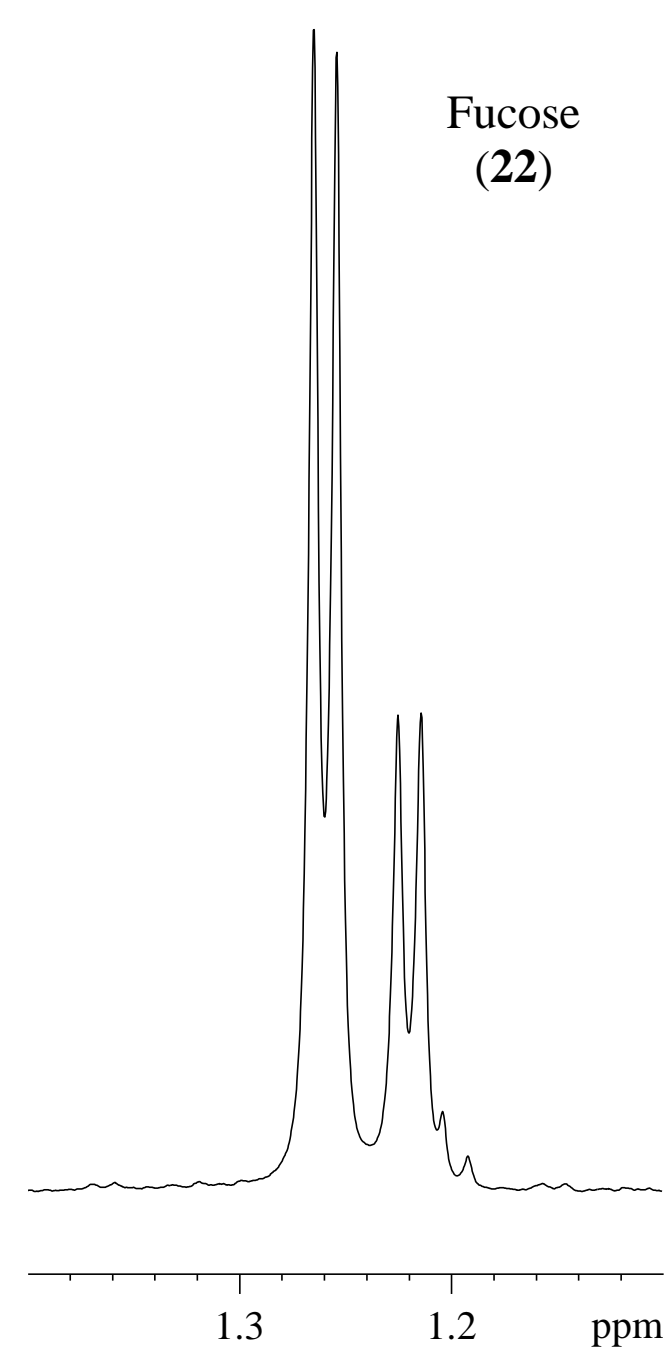

6-Deoxy Sugar Methyl Signals

$\left(600 \mathrm{MHz}{ }^{1} \mathrm{H}-\mathrm{NMR} ; 2 \mathrm{M} \mathrm{D}_{2} \mathrm{SO}_{4} / \mathrm{D}_{2} \mathrm{O} ; 30^{\circ} \mathrm{C}\right)$

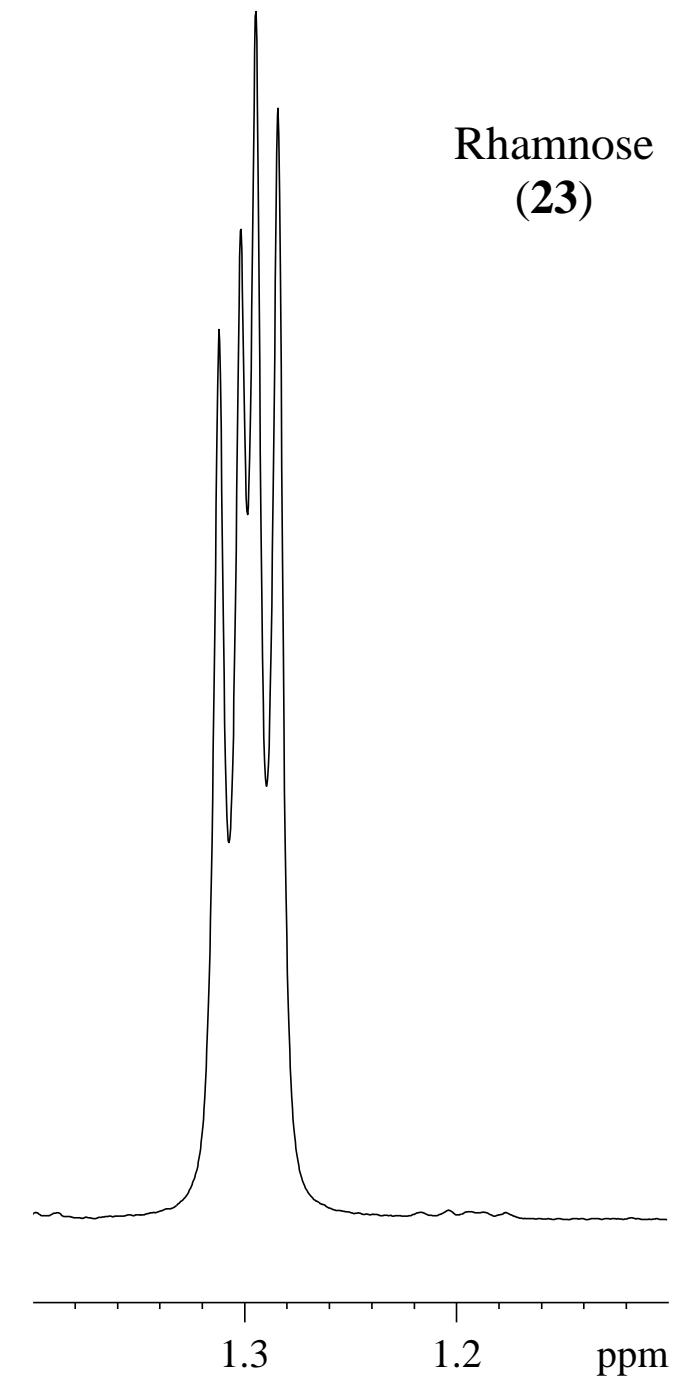

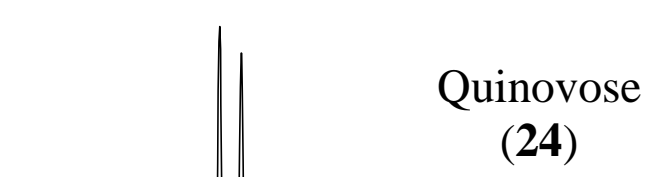

(24) 
Figure S3

Furfural Signals

(600 MHz ${ }^{1} \mathrm{H}-\mathrm{NMR} ; 2 \mathrm{M} \mathrm{D}_{2} \mathrm{SO}_{4} / \mathrm{D}_{2} \mathrm{O} ; 30^{\circ} \mathrm{C}$ )

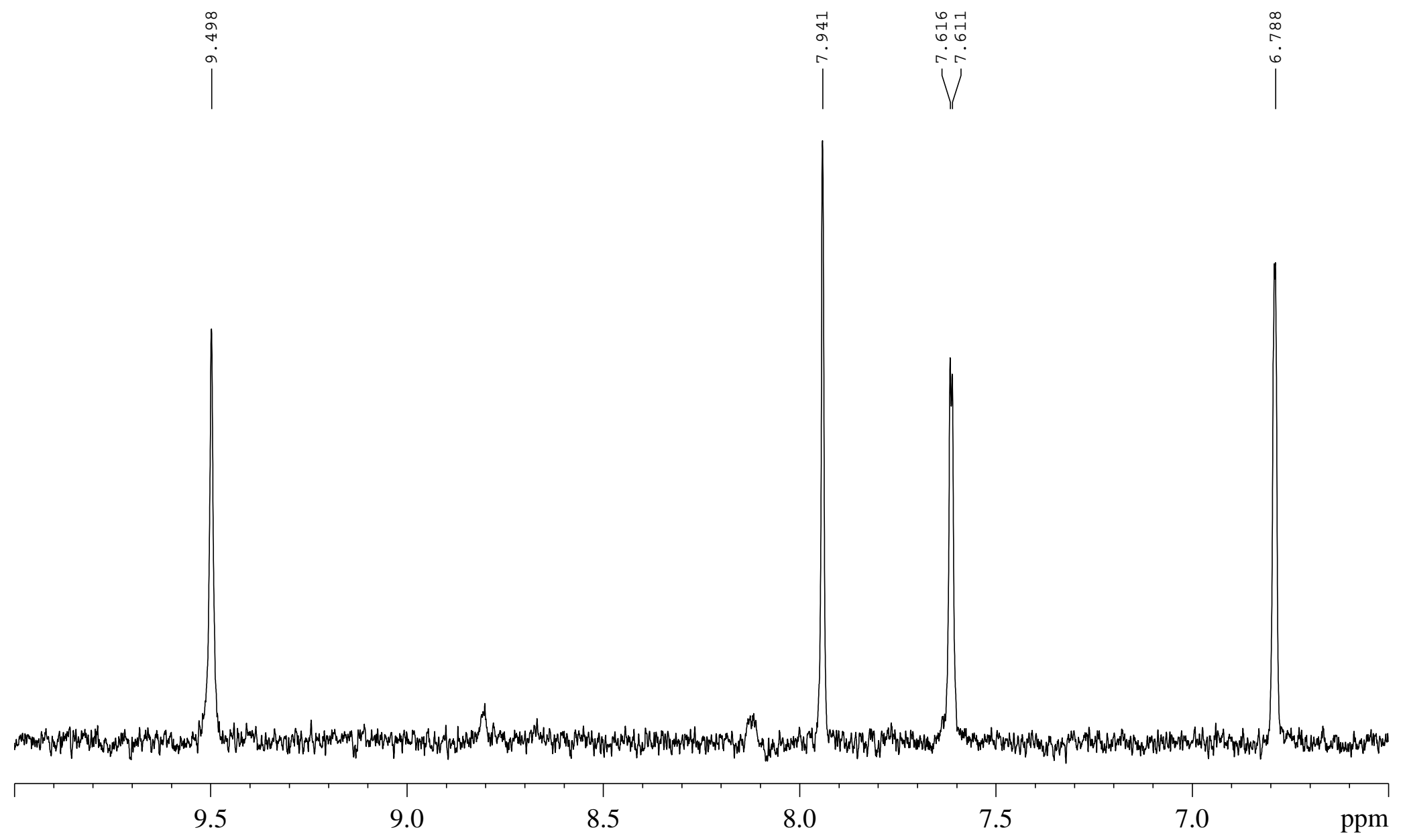


Figure S4

Timecourse at $100^{\circ} \mathrm{C}$

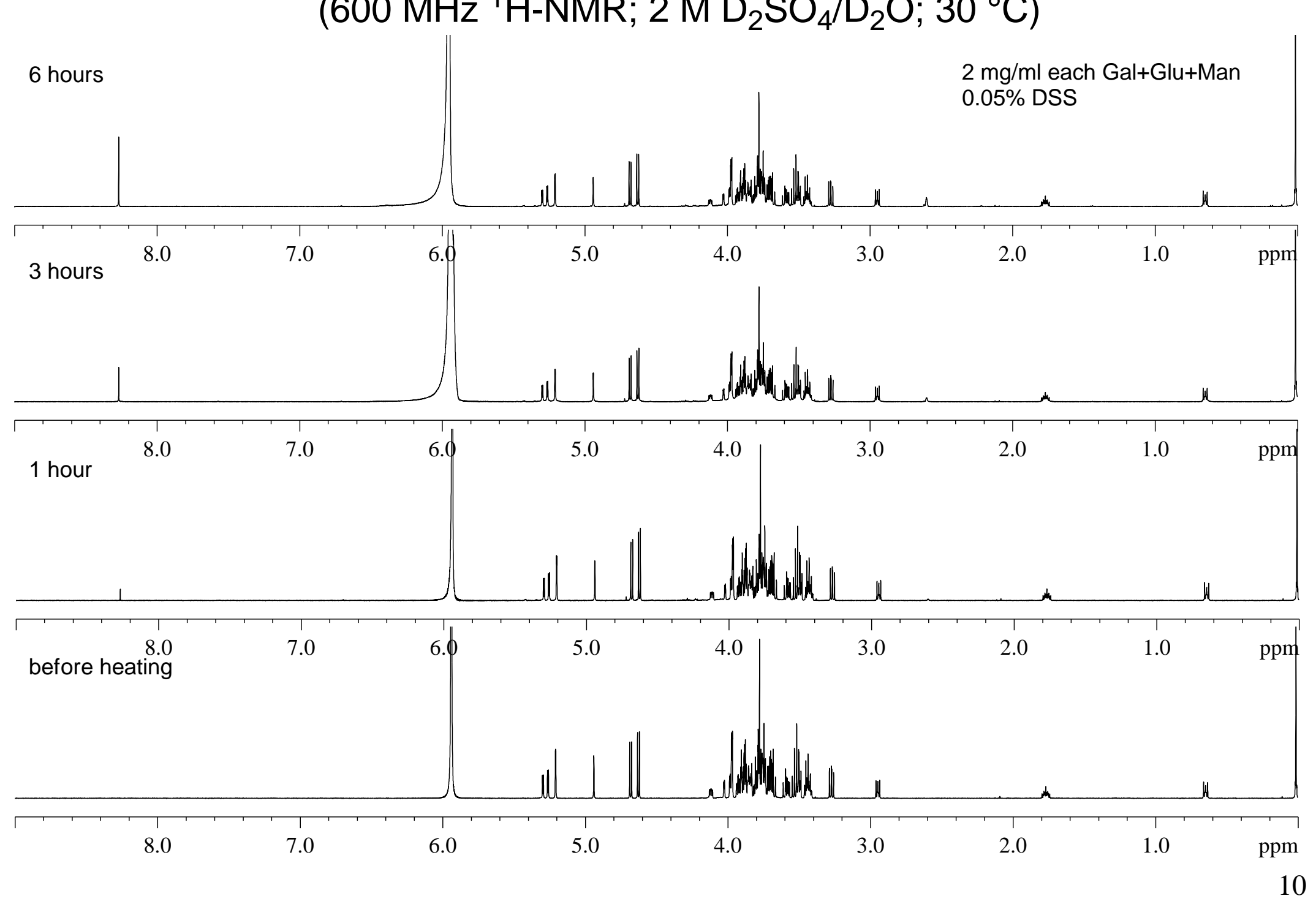


Figure S5

Concentration Dependence
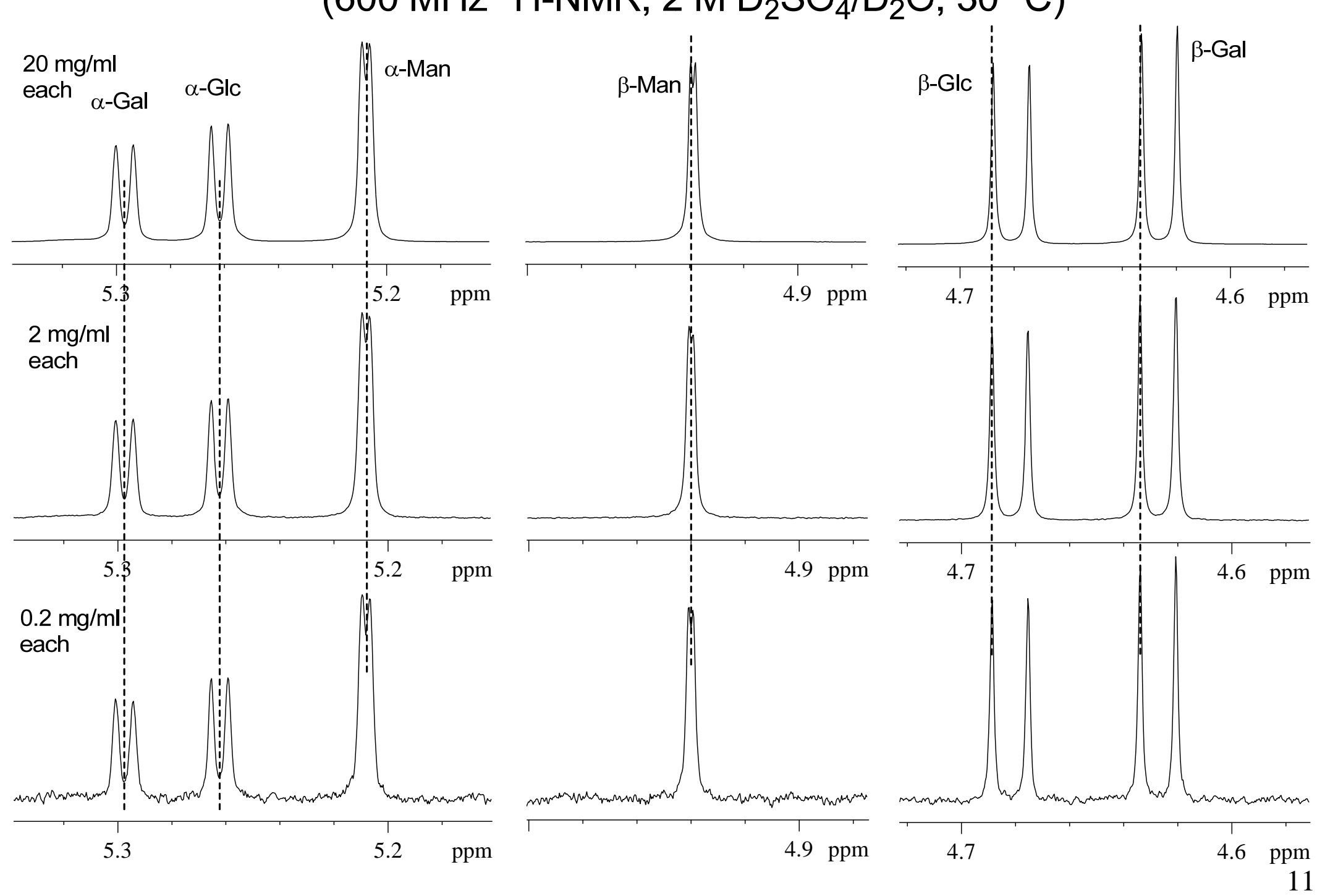
Figure S6 Glucose at Varying Acid Concentrations

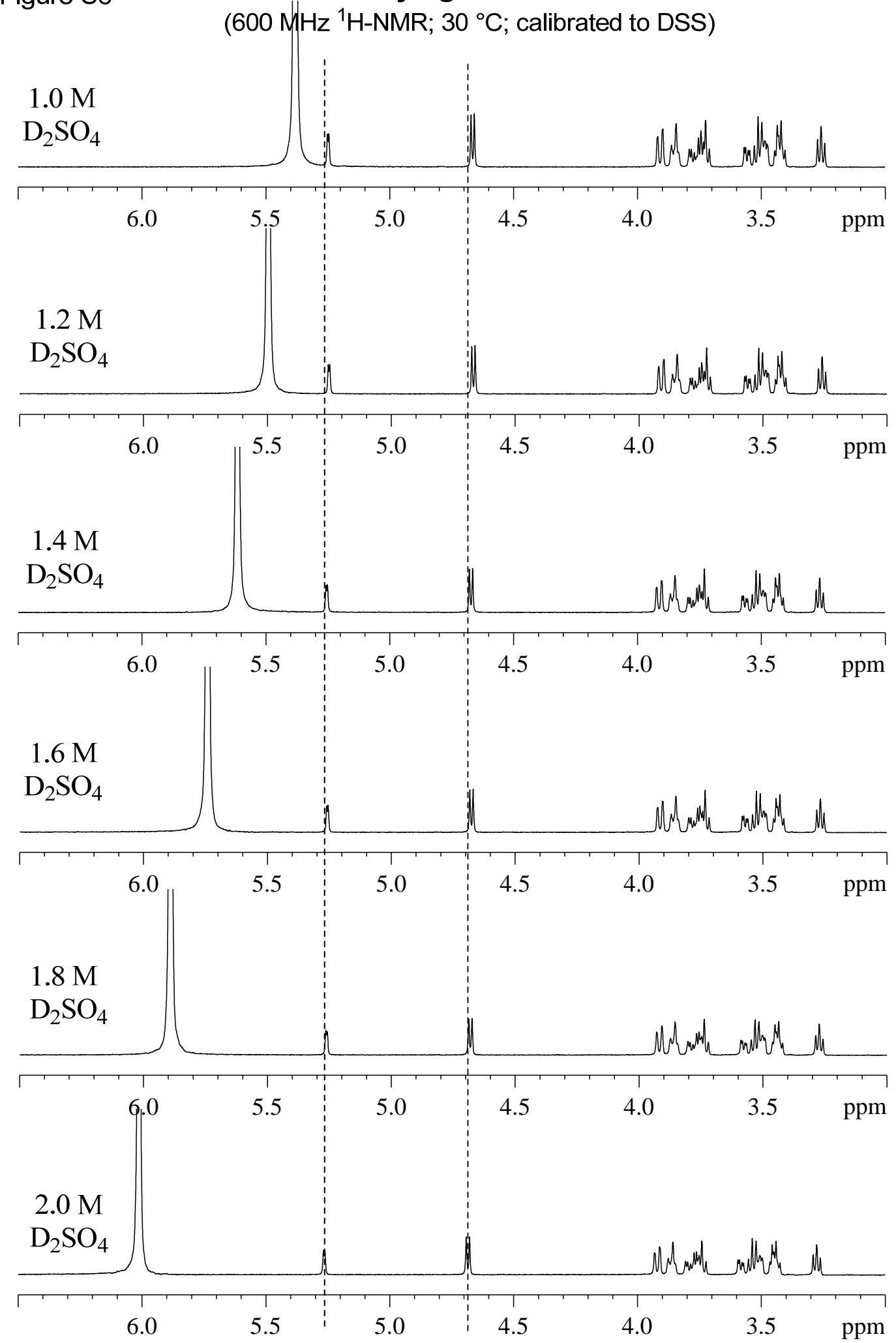




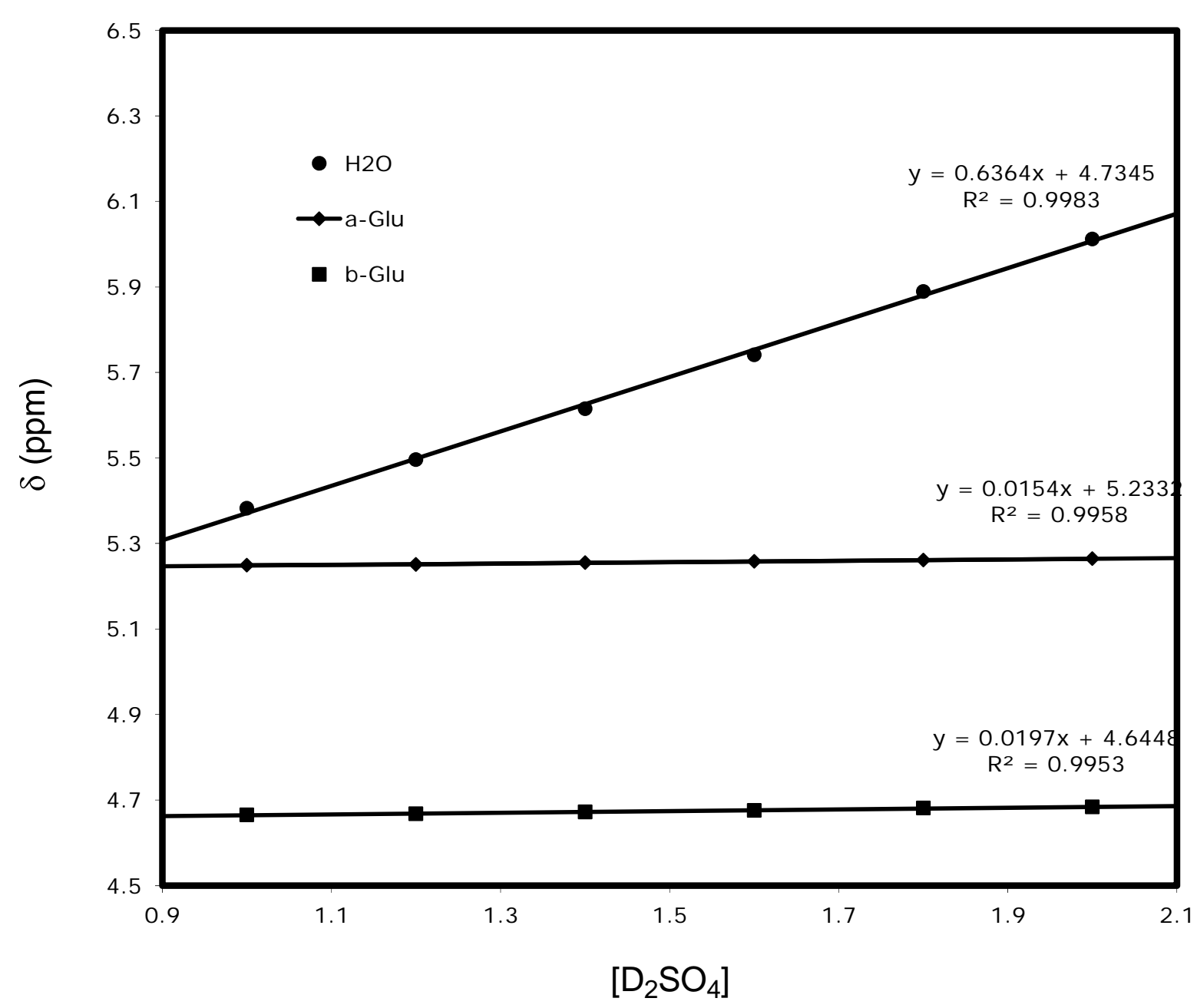


Figure S8

T1 Analysis

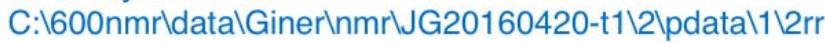

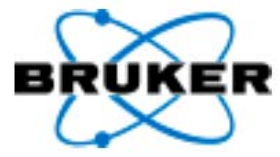

\begin{tabular}{|l|l|}
\hline Fitted function: & $\mathrm{f}(\mathrm{t})=10^{*}\left[1-2^{*} \exp (-\mathrm{t} / \mathrm{T} 1)\right]$ \\
\hline Random error estimation of data: & RMS per spectrum (or trace/plane) \\
\hline Systematic error estimation of data: & worst case per peak scenario \\
\hline Fit parameter Error estimation method: & from fit using arbitray y uncertainties \\
\hline Confidence level: & $95 \%$ \\
\hline Used peaks: & \\
\hline Used integrals: & area integral \\
\hline Used Mixing time: & all values (including replicates) used \\
\hline
\end{tabular}

\begin{tabular}{|l|l|l|l|}
\hline \multicolumn{1}{|c|}{ Peak name } & \multicolumn{1}{c|}{ T1 [s] } & \multicolumn{1}{c|}{ error } \\
\hline 1 & 5.305 & 1.75 & 0.05662 \\
\hline 2 & 5.269 & 1.79 & 0.05058 \\
\hline 3 & 5.229 & 2.00 & 0.02674 \\
\hline 4 & 5.214 & 1.91 & 0.05385 \\
\hline 5 & 4.948 & 1.36 & 0.07823 \\
\hline 6 & 4.690 & 1.30 & 0.02596 \\
\hline 7 & 4.642 & 1.38 & 0.04638 \\
\hline 8 & 4.613 & 1.84 & 0.03485 \\
\hline
\end{tabular}


Figure S8

T1 Analysis

C:1600nmrldatalGiner/nmrlJG20160420-t112lpdatal112rr

BRUKER

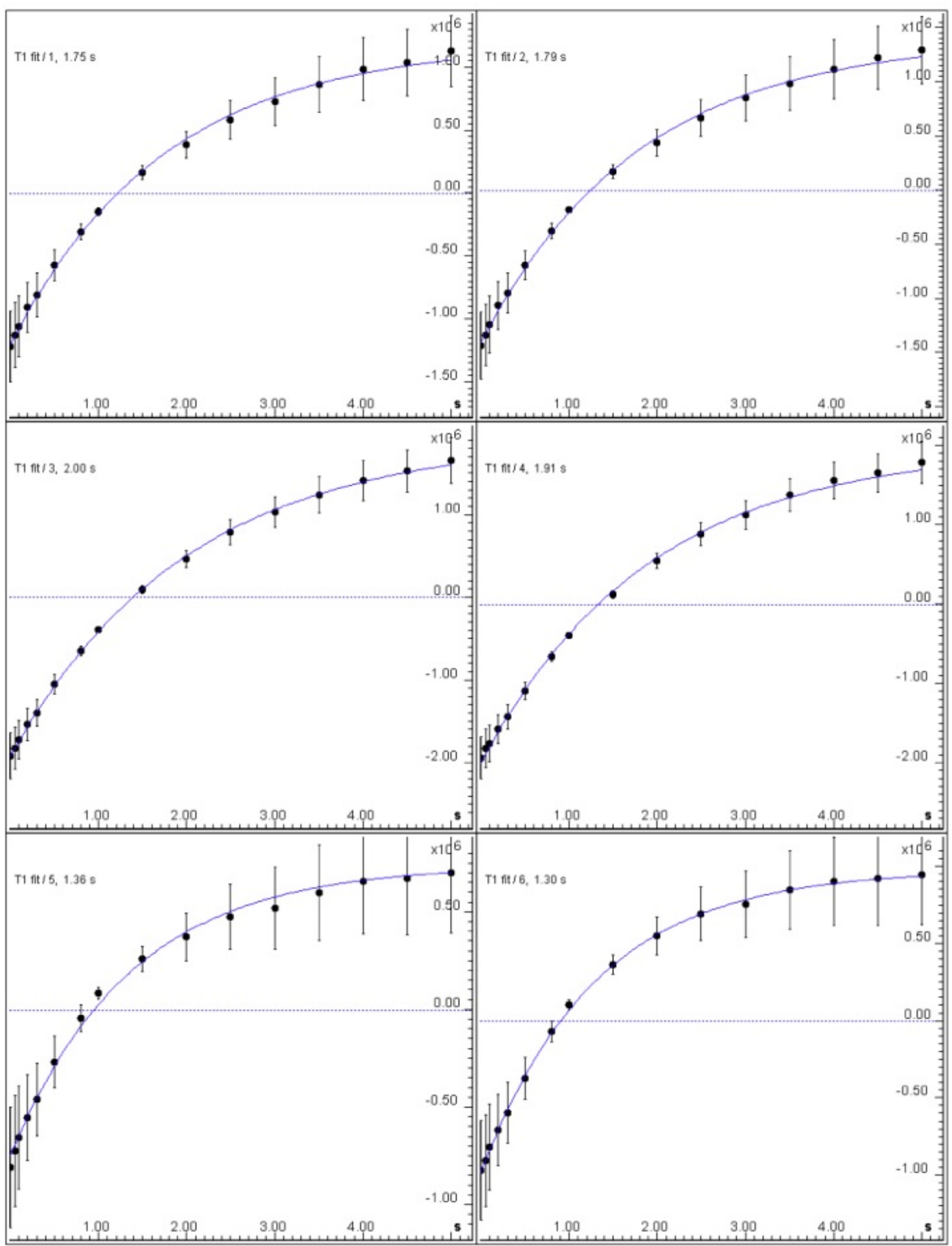


Figure S8

T1 Analysis

C:I600nmrldatalGiner/nmrlJG20160420-t1\2/pdatal1/2rr
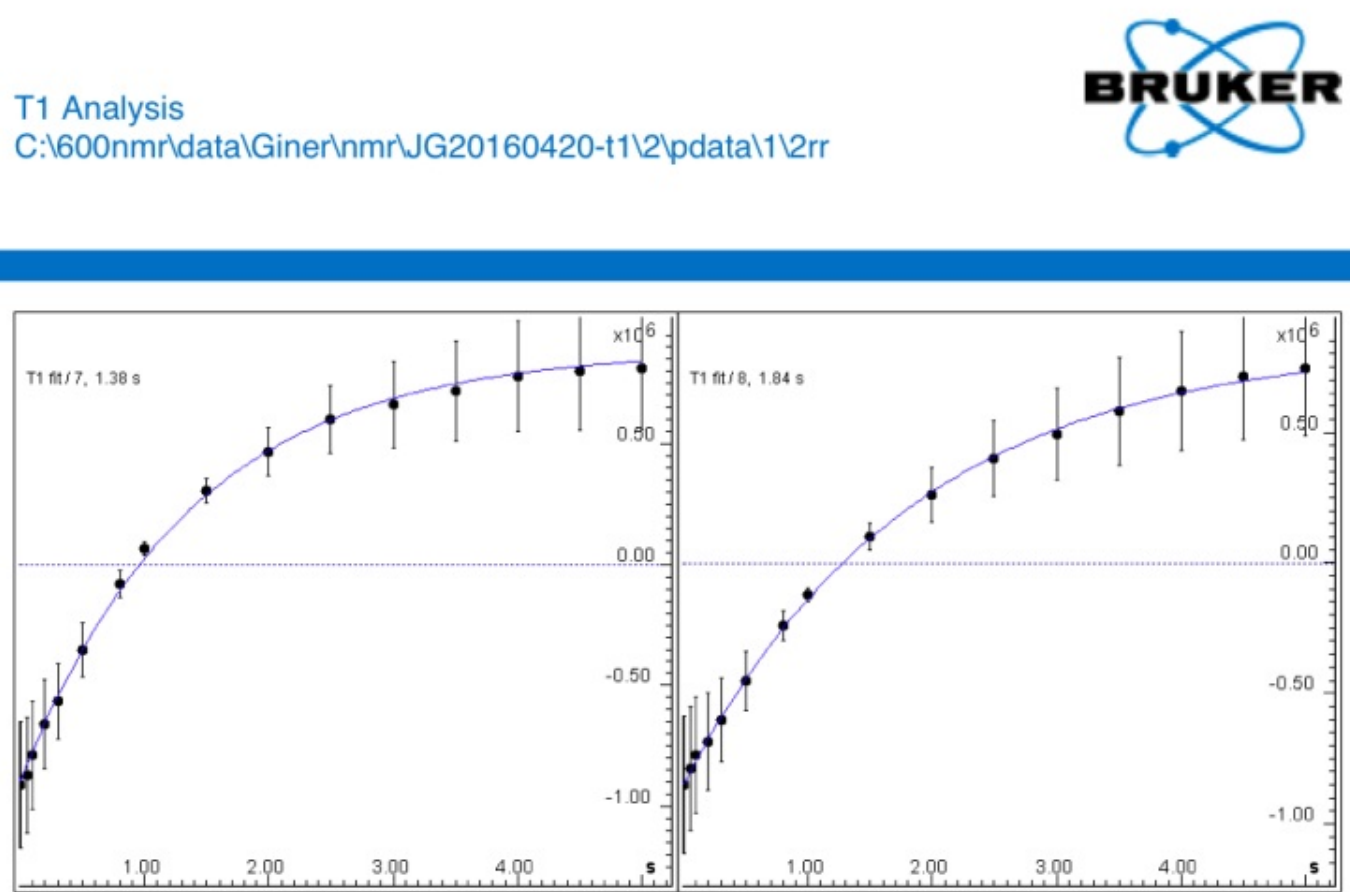
Figure S9

\section{Naringin Hydrolysis in 2M $\mathrm{D}_{\mathbf{2}} \mathrm{SO}_{4} / \mathrm{DMSO}$ 1:9}

(600 MHz ${ }^{1} \mathrm{H}-\mathrm{NMR} ; 30{ }^{\circ} \mathrm{C}$; calibrated to DSS)

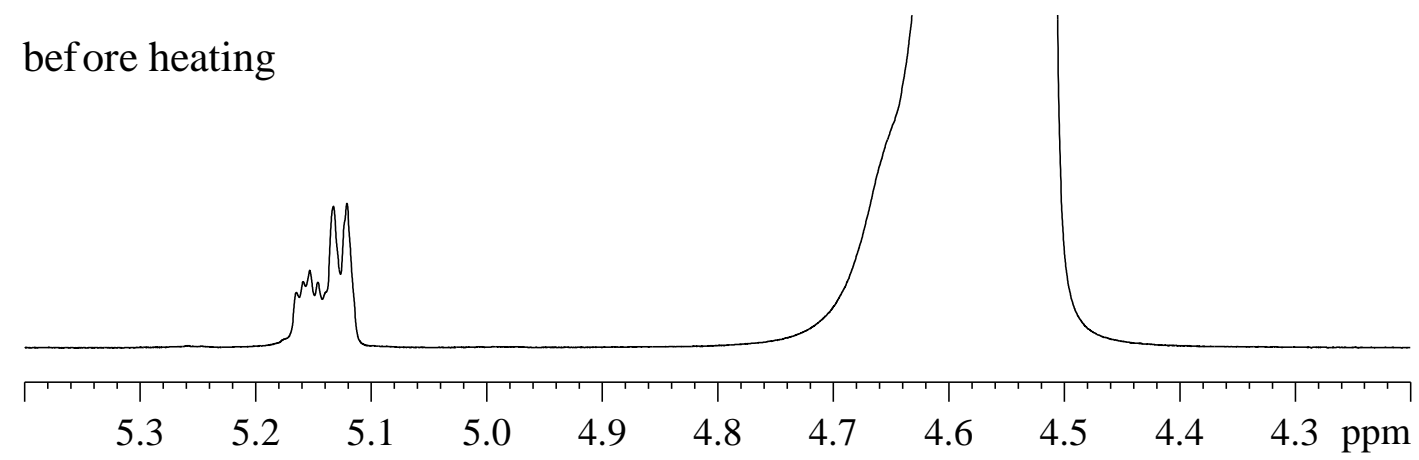

$90{ }^{\circ} \mathrm{C} 40 \mathrm{~min}$

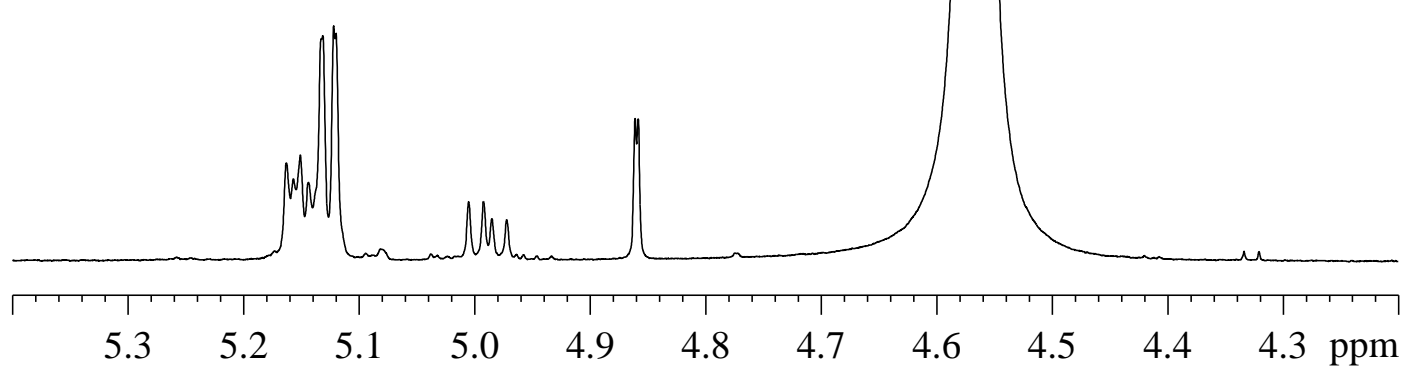

$90{ }^{\circ} \mathrm{C} 3$ hr $40 \mathrm{~min}$

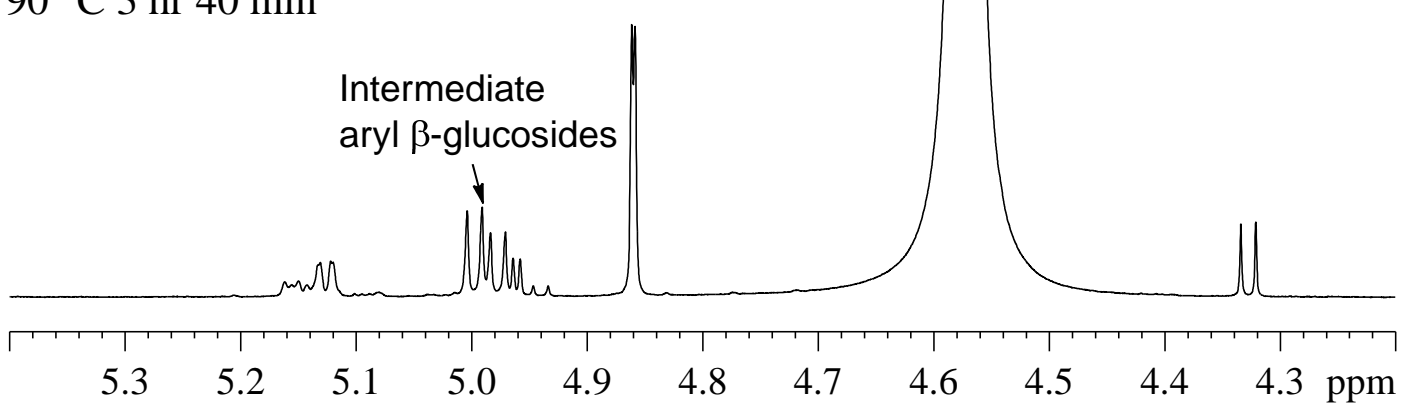

$90{ }^{\circ} \mathrm{C} 19 \mathrm{hr}$

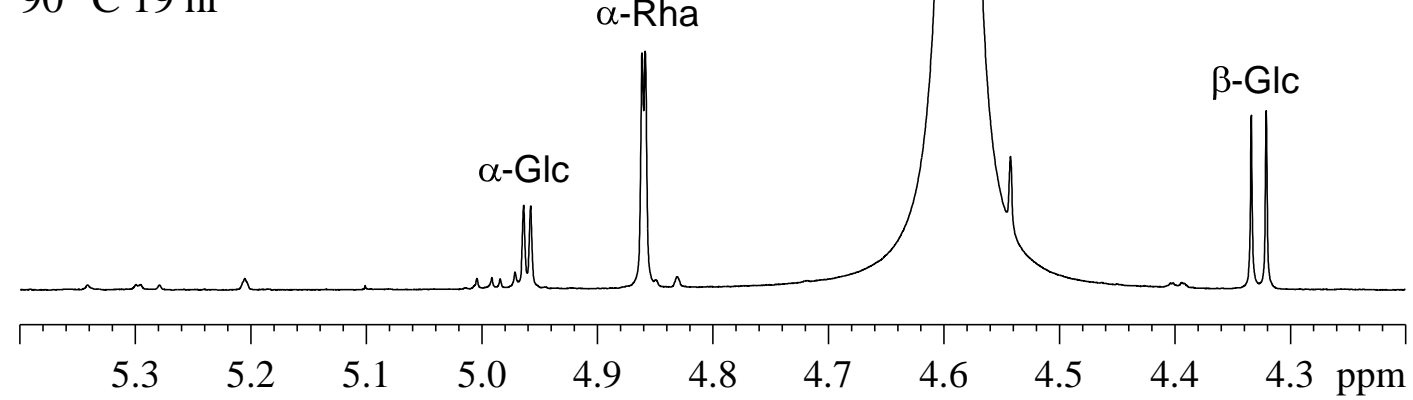


Figure S10

Glucose at Varying DMSO Concentrations

(600 MHz ${ }^{1} \mathrm{H}-\mathrm{NMR} ; 30^{\circ} \mathrm{C}$; calibrated to DSS)

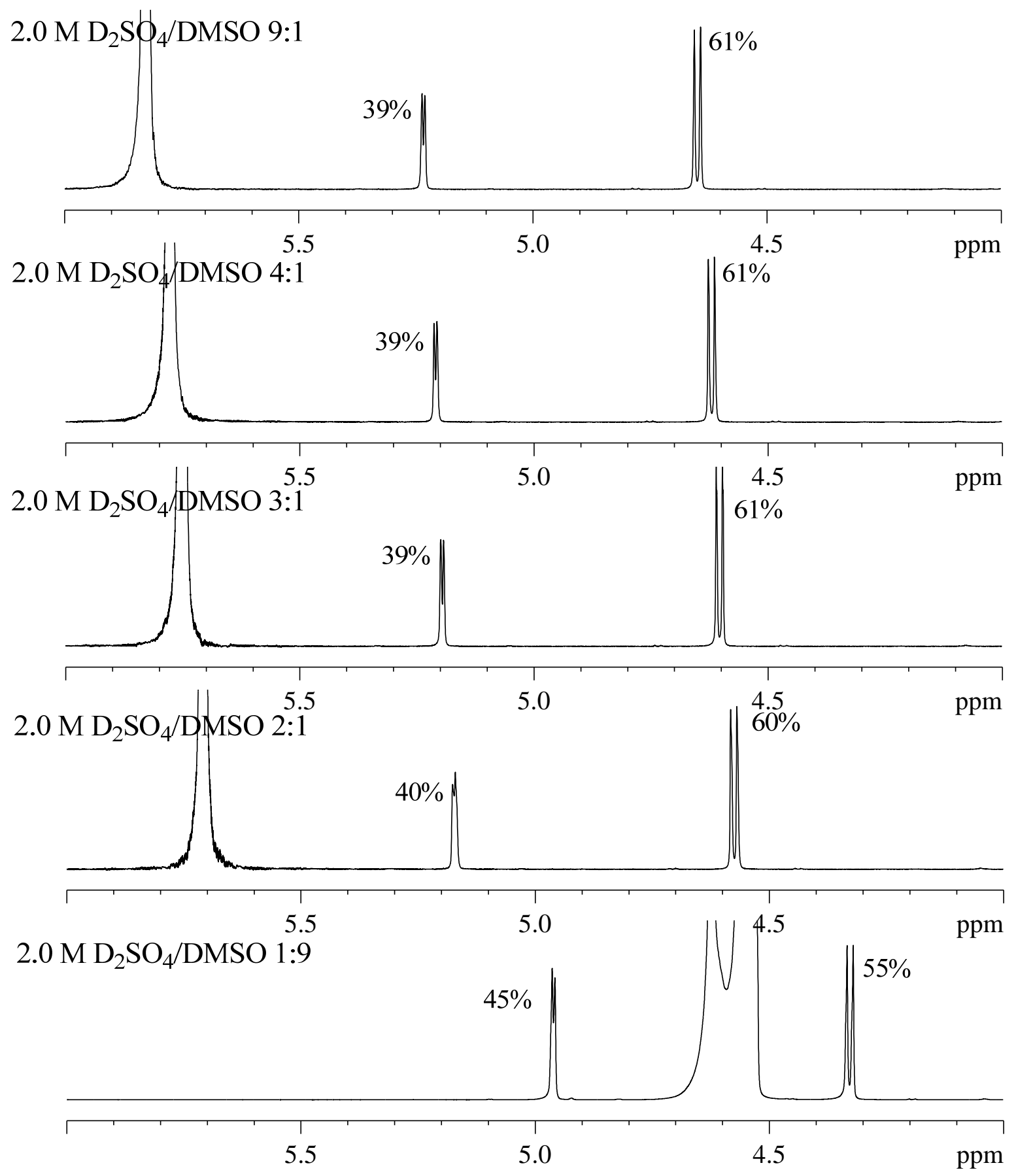

